\title{
Spectra of Field Fluctuations in Braneworld Models with Broken Bulk Lorentz Invariance
}

\author{
Peter Koroteev ${ }^{\ddagger * *, \star}$ And Maxim Libanov $\ddagger$ \\ ${ }^{\ddagger}$ Institute for Nuclear Research of the Russian Academy of Sciences \\ 60th October Anniversary Prospect 7a, Moscow 117312, Russia \\ ${ }^{*}$ Institute for Theoretical and Experimental Physics \\ B. Cheremushkinskaya 25, Moscow 117218, Russia \\ *University of Minnesota, School of Physics and Astronomy \\ 116 Church Street S.E. Minneapolis, MN 55455, USA \\ koroteev@physics.umn.edu, ml@ms2.inr.ac.ru
}

\begin{abstract}
We investigate five-dimensional braneworld setups with broken Lorentz invariance continuing the developments of [1], where a family of static self-tuning braneworld solutions was found. We show that several known braneworld models can be embedded into this family. Then we give a qualitative analysis of spectra of field fluctuations in backgrounds with broken Lorentz invariance. We also elaborate on one particular model and study spectra of scalar and spinor fields in it. It turns out that the spectra we have found possess very peculiar and unexpected properties.
\end{abstract}




\section{Introduction and Overview}

In modern cosmology and particle physics we are faced with several problems like the cosmological constant problem and the hierarchy problem. Some time ago the novel braneworld programme was proposed $[2-4]$ which made the above problems more tractable [5] (see also [6] for string theoretical analysis). In this treatment our Universe is represented as a fourdimensional submanifold (a brane) embedded into a manifold of higher dimension (a bulk). The presence of extra dimensions changes cosmological evolution $[7]$ and could be made manifest at forthcoming high energy experiments $[8]$ and in the other measurements $[9]$. Among an enormous number of the large extra dimensional models the Randall-Sundrum models (RS1 [3] and the RS2 [4]) appeared to be very successful, as they allow one to look at the above two problems from a new viewpoint without spoiling four-dimensional physics at observable distances and energies. The RS models have initiated the extended and prospective programme of large extra dimensional physics; it is not easy to present an exhaustive list of publications devoted to physics in extra dimensional setups, one can use various reviews and notes for finding necessary literature $[10]$.

In the Randall-Sundrum models the five dimensional anti de-Sitter space is used as a bulk filled by the negative cosmological constant and one (RS2) or two (RS1) branes represented by $3+1$ dimensional hypersurfaces embedded into the bulk. One can also consider various generalizations of this configuration and study their phenomenological manifestations. One of the possible extensions of the RS models consists of an inclusion of an energy density (matter) in consideration which is somehow distributed in the bulk and/or on the brane. The presence of matter may lead in general to breaking of Lorentz invariance. Different aspects of Lorentz invariance violation in models with large extra dimensions were considered in $[?, 11]$. In these models Lorentz invariance was broken spontaneously on the brane due to the presence of a symmetry-breaking potential. However, one can also consider models with broken Lorentz invariance in the bulk and study deviations from the four-dimensional physics on the brane which a priori preserves Lorentz invariance (see, e.g., [12,13,1]). It is worth noting however that, as it was proved in [1], the matter located in the bulk which produces a Lorentz violated static smooth metric, inevitably violates the null energy conditions.

In the present work we investigate field fluctuations in the backgrounds found in [1], where the bulk metric explicitly breaks Lorentz invariance and has the following form

$$
d s^{2}=\mathrm{e}^{-2 a(z)} d t^{2}-\mathrm{e}^{-2 b(z)} d \mathbf{x}^{2}-d z^{2} .
$$

Here $(t, \mathbf{x})$ are four-dimensional coordinates parallel to the brane and $z$ is the coordinate along the extra dimension. In [1] a two-parametric family of solutions of the bulk Einstein equations has been found in the presence of an ideal anisotropic relativistic fluid in the bulk. ${ }^{1}$ The functions $a$ and $b$ from (1.1) are linear functions of $z$

$$
a(z)=\xi k|z|, \quad b(z)=\zeta k|z|, \quad k>0,
$$

with the coefficients $\xi$ and $\zeta$ related to the anisotropy parameters of the ideal fluid. In this paper we introduce a space parametrized by $\xi$ and $\zeta$ which describes different metric tensors and study how the fluctuation spectra of fields behave at different values of $\xi$ and $\zeta$.

\footnotetext{
${ }^{1}$ In accordance with the theorem proved in 1, this fluid violates null energy conditions.
} 
First, we perform more generic analysis of the spectra employing the metric (1.1) without specifying the functions $a$ and $b$. One of our main results is that the spectrum pattern is governed by an asymptotic behavior of the functions $a$ and $b$. If $a(z) / b(z) \rightarrow 0$ as $z \rightarrow \infty$ then the spectrum is discrete and all excitation modes are localized in some vicinity of the brane located at $z=0$. The reason of the localization is that in this case the potential responsible for the localization grows away from the brane. In the opposite case, if $a(z) / b(z)$ does not vanish at infinity, then at least part of the spectrum becomes continuous and excitation modes with energy high enough delocalize from the brane. In this case the potential tends to some constant value (or vanishes) far away from the brane. We further embed some known models: flat Minkowski space, the RS2 model [4], Dubovsky model [12], into our parametric solution.

In addition, we elaborate on a new model with the metric (corresponding to $\xi=0, \zeta=1$ in $(1.2))$

$$
d s^{2}=d t^{2}-\mathrm{e}^{-2 k|z|} d \mathbf{x}^{2}-d z^{2},
$$

where $g_{00}$ metric coefficient is unwarped, and $g_{i j}$ coefficients are warped in the same way as in the RS mode $]^{2}$. The model with metric $(1.3)$ is shown to have dramatically different properties than the models $[4,12]$ as long as localization of perturbations and speed of particle propagation are considered. In the former field perturbations are localized near the brane and their spectrum is discrete, while for the latter there may exist only several (quasi-)localized modes and the spectrum is continuous. We study spectra of scalar and spinor excitations in the background (1.3). In both cases we find that there is a zero (or gapless) mode - a state with vanishing energy as three-dimensional momentum tends to zero. From the fourdimensional point of view a zero mode corresponds to a massless state and this is the only state which presents in the low energy effective theory. It is remarkable that in spite of the metric $(1.3)$ is Lorentz invariant on the brane $(z=0)$ the zero mode dispersion relation explicitly breaks Lorentz invariance at low momenta because of the "tail" of the zero mode wave function "feels" the breaking of Lorentz invariance in the bulk.

The paper is organized as follows. In Sec. 2 we make some qualitative remarks about field fluctuation spectra in different backgrounds with broken bulk Lorentz invariance. In Sec. 3 and Sec. 4 we investigate spectra of scalar and spinor fields respectively in these backgrounds. Our conclusions and final comments are given in Sec. 5. Detailed derivation of the fermionic spectrum is presented in App. A.

\section{Localization and Delocalization of Fluctuations}

In this section we show that the asymptotic behavior of the functions $a$ and $b$ in $(1.1)$ is responsible for the pattern of perturbation spectra of fields in the backgrounds in question. In the next sections we investigate how different perturbations behave in a particular example. From qualitative description one can understand something about those perturbations in a generic fashion.

\footnotetext{
${ }^{2}$ Five-dimensional coordinates $x^{A}$ are labeled by capital Latin indices $0,1,2,3,5$; three-dimensional coordinates $x^{i}$ are labeled by lower Latin indices $1,2,3$.
} 
Considering some bulk matter field one can think of (quantum) particles propagating in the bulk. Let us assume that our model admits semi-classical approximation. If so, then particles do not considerably deviate from their classical trajectories and become "localized" in domains with higher density of these trajectories. Thus investigation of a geodesic configuration helps us in understanding of localization properties of field fluctuations. For simplicity we consider a massless free field, so the corresponding particles propagate along null geodesics. A geodesic is a solution to the following equation

$$
\ddot{x}^{A}+\Gamma_{B C}^{A} \dot{x}^{B} \dot{x}^{C}=0
$$

where $\Gamma_{B C}^{A}$ are Christoffel symbols for the metric $(1.1)$ and the dots above $x^{A}$ stand for derivatives with respect to the affine parameter. Equations for the 0,1,2,3 components can be integrated straightforwardly

$$
\dot{x}^{0}=\alpha \mathrm{e}^{2 a}, \quad \dot{x}^{i}=\beta^{i} \mathrm{e}^{2 b}, \quad i=1,2,3,
$$

where $\alpha$ and $\beta^{i}$ are the integration constants. Using this fact and light cone equation for the massless particle $g_{A B} \dot{x}^{A} \dot{x}^{B}=0$ one finds the particle velocity along fifth direction

$$
\dot{z}^{2}=\alpha^{2} \mathrm{e}^{2 a}-\left(\beta^{i}\right)^{2} \mathrm{e}^{2 b} .
$$

It follows from this equation that if the three-dimensional initial velocity vanishes, $\beta_{i}=0$, then the particle motion is always non-finite. From the quantum mechanical point of view it means that the particle with zero three-dimensional momentum cannot be localized near the brane. The particle motion is also non-finite in the Lorentz invariant case, $a(z)=b(z)$. It does not, however, contradict the fact of presence of a zero mode in the RS2 setup - the semi-classical description is certainly not applicable for a zero mode.

Let us consider the case $a(z) \rightarrow+\infty$ and $b(z) \rightarrow+\infty$ as $z \rightarrow \infty$. This is the most general case corresponding to the finite volume extra dimension. In this case if $a(z) / b(z) \rightarrow 0$ as $z \rightarrow \infty$ then the particle motion is finite $\left(\beta_{i} \neq 0\right)$ which corresponds to localization of the quantum particle in some domain near the brane. The opposite case $a(z) / b(z) \rightarrow \infty$ or $a(z) / b(z) \rightarrow$ const as $z \rightarrow \infty$ corresponds to the delocalization regime and there exists a probability of a detection of the particle far away from the brane.

We would like to mention again that the considerations used in this section are merely qualitative. An advantage of this description is that its conclusions are universal and can be applied to any field theory in the bulk.

\section{Scalar Fluctuations}

After qualitative analysis in the previous section let us proceed to more specific treatment and investigate fluctuations of a scalar field in the bulk. For simplicity in this section we consider massless scalar field. First, we make a qualitative description of how the perturbations behave for generic form of the metric (1.1) and discuss some special points in the space parameterized by $\xi$ and $\zeta$. In the end we elaborate on the spectrum of the scalar field in the background (1.3). 
Let us consider the massless scalar field with the five-dimensional action

$$
S=\int d t d \mathbf{x} \int_{-\infty}^{+\infty} d z \sqrt{g} g^{A B} \partial_{A} \phi \partial_{B} \phi
$$

We assume that presence of the field $\phi$ in the bulk does not affect the background metric. The equation of motion reads

$$
\left[-\partial_{z}^{2}+\left(a^{\prime}+3 b^{\prime}\right) \partial_{z}+\mathrm{e}^{2 a(z)} \partial_{t}^{2}-\mathrm{e}^{2 b(z)} \partial_{i}^{2}\right] \phi=0,
$$

and, after four-dimensional Fourier transform

$$
\phi(t, \mathbf{x}, z)=\frac{1}{(2 \pi)^{2}} \int d E d \mathbf{p} \mathrm{e}^{i E t-i \mathbf{p x}} \phi(E, \mathbf{p}, z),
$$

it takes the following form

$$
\left[\partial_{z}^{2}-\left(a^{\prime}+3 b^{\prime}\right) \partial_{z}+E^{2} \mathrm{e}^{2 a(z)}-p^{2} \mathrm{e}^{2 b(z)}\right] \phi=0,
$$

where we denoted $p=\sqrt{\mathbf{p}^{2}}$. For convenience we introduce another function

$$
\chi(z)=\exp \left(-\frac{1}{2} a-\frac{3}{2} b\right) \phi(z),
$$

and the above equation can be rewritten as follows

$$
\left[\partial_{z}^{2}+E^{2} \mathrm{e}^{2 a(z)}-p^{2} \mathrm{e}^{2 b(z)}+\frac{a^{\prime \prime}+3 b^{\prime \prime}}{2}-\frac{\left(a^{\prime}+3 b^{\prime}\right)^{2}}{4}\right] \chi=0 .
$$

We also introduce the new coordinate $y$ by the relations

$$
\frac{\partial}{\partial z} y(z)=\mathrm{e}^{a(z)}, \quad y(z)=\int_{0}^{z} \mathrm{e}^{a(\rho)} d \rho
$$

In terms of this new coordinate, the equation (3.1) takes the following form

$$
\frac{\partial^{2} \chi}{\partial y^{2}}+\left(E^{2}-V\right) \chi=0
$$

where

$$
V=-\frac{1}{4}\left(\frac{\partial a}{\partial y}\right)^{2}+\frac{9}{4}\left(\frac{\partial b}{\partial y}\right)^{2}+p^{2} \mathrm{e}^{2(b-a)}-\frac{1}{2}\left(\frac{\partial^{2} a}{\partial y^{2}}+3 \frac{\partial^{2} b}{\partial y^{2}}\right) .
$$

Let us consider the potential $V$ in more detail. In what follows we assume $\mathbb{Z}_{2}$ bulk reflection symmetry $z \rightarrow-z$ with respect to the brane. It means in particular that the functions $a$ and $b$ in (1.1) depend on the absolute value of $z$ (and, hence, on $y$ ) only. The potential $V$ is bilinear in the first and linear in the second derivatives, hence in general it has a delta-function term emerging from the last term in (3.3). The sign of the last term can be either positive or negative depending on the behavior of the functions $a$ and $b$ in the vicinity 
of the origin. If the sign is positive then we have a delta-well in the potential and a delta-peak otherwise. If Lorentz invariance is not broken $(a(y)=b(y))$ then the momentum-dependent term is merely a constant and does not affect the shape of the potential $V$. However, if this is not the case, depending on the difference $b-a$ in the exponent the shape of the potential can be drastically modified. If $b>a$ as $y$ increases into the bulk, then the momentum-dependent term increases as well and soon will prevail the other terms ${ }^{3}$ The opposite configuration with $b<a$ makes (far enough from the brane) the momentum-dependent term negligible compared to the others. Thus we see that the generic potential (3.3) can be classified by the following properties

- Behavior at infinity which is controlled by the Lorentz invariance violation (the sign of $b-a$ ). The potential $V$ increases/decreases as $y \rightarrow \infty$.

- Sign of the delta-function term. The potential may have either delta-well or delta-peak at the origin depending on this sign.

This rough classification enables us to describe the behavior of fluctuations in each case.

- If the momentum-dependent term increases as $y \rightarrow \infty$ the potential has a box-type shape and the excitation spectrum is discrete. The potential may have local minima and maxima, but qualitative pattern of the spectrum is determined by its behavior at infinity. On the contrary, if the potential decays at infinity, then we have continuous spectrum of plane waves propagating along $y$-direction. Some combination of these two scenarios is possible if $V \rightarrow V_{\infty}=$ const as $z \rightarrow \infty$. Then those modes with $E^{2}<$ $V_{\infty}$ belong to discrete spectrum and modes with $E^{2}>V_{\infty}$ contribute to continuous spectrum.

- The sign of the delta-function term affects zero mode existence. In a delta-well there might be a zero-mode $\epsilon^{4}$ and its existence is very unlikely in a configuration with a delta-peak.

Unfortunately rigorous analysis of (3.2) with arbitrary functions $a$ and $b$ is troublesome as the corresponding equations cannot be solved analytically. Nevertheless, the qualitative behavior of perturbations is not changed if we consider metrics, different in general, but having similar form in the vicinity of the origin and at infinity. In Sec. 3.2 we choose the functions $a$ and $b$ to be of the form (1.2) which enables us to obtain exact solutions for certain configurations.

\subsection{Parameter Space}

In [1] the static solution for the ansatz $(1.1)$ has been found in the presence of an anisotropic perfect fluid in the bulk. The metric has the following form

$$
d s^{2}=\mathrm{e}^{-2 \xi k|z|} d t^{2}-\mathrm{e}^{-2 \zeta k|z|} d \mathrm{x}^{2}-d z^{2} .
$$

\footnotetext{
${ }^{3}$ We do not pay much attention here on rigorous analysis of conditions how the functions $a$ and $b$ affect the potential, leaving it to the next subsection where an explicit example is elaborated.

${ }^{4}$ There might be also no zero mode, but one cannot understand whether it exists or not without detailed analysis.
} 
Obviously, the chosen parametrization with $\xi, \zeta$ and $k$ is overcomplete,,$^{5}$ one of the parameters can be scaled away. Nevertheless, in what follows we keep all three parameters unconstrained in order to simplify further analysis.

One can depict the $(\xi, \zeta)$ parameter space as shown in Fig. 1. One has flat Minkowski

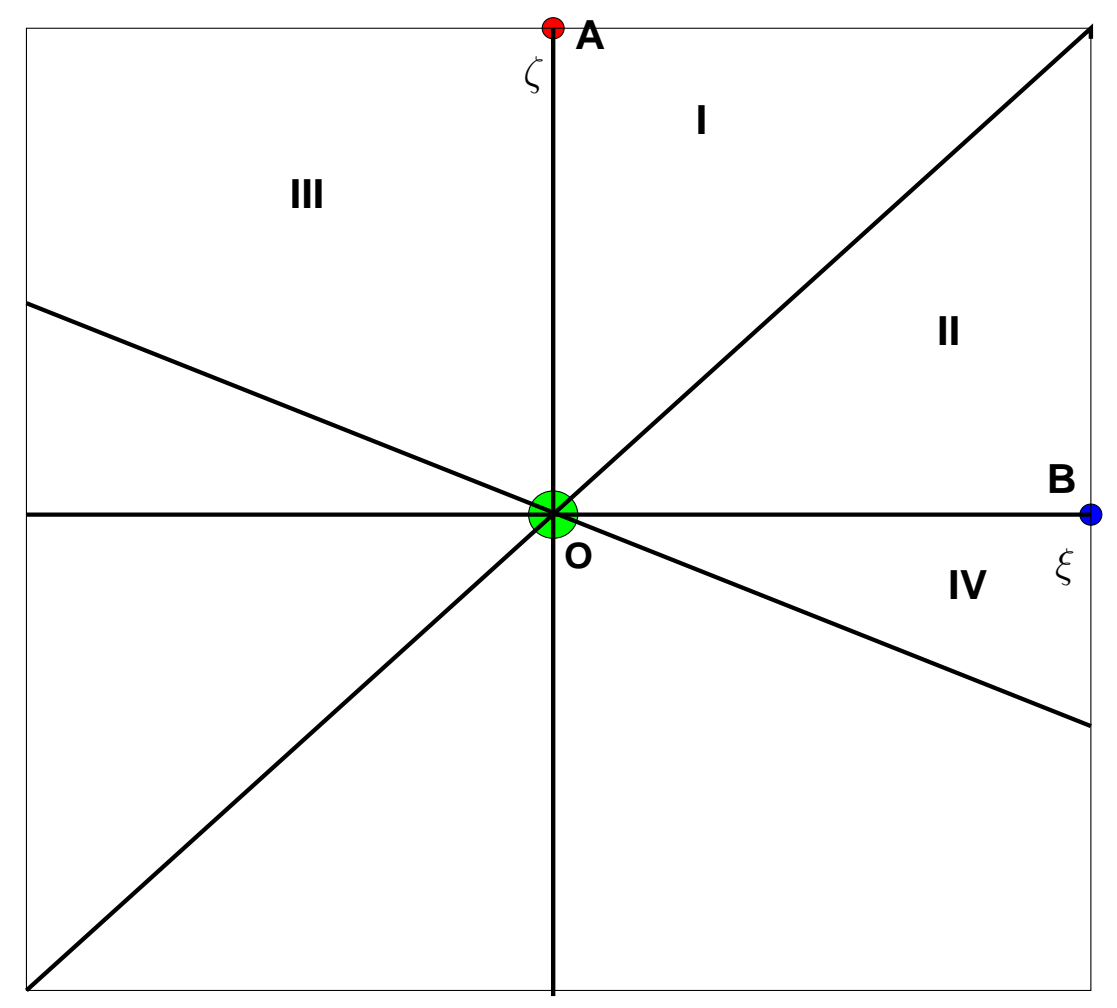

Figure 1: The parameter space. We discuss our theory in the following subsets of this square: Minkowski (the origin O), $A d S_{5}$ (the diagonal), type I (upper triangle) and type II (lower triangle).

metric at the origin $\mathrm{O}, A d S_{5}$ metric on the main diagonal, the models with $\xi<\zeta$ (type II models) in the lower triangle and models with $\xi>\zeta$ (type I models) in the upper triangle. For instance, the RS2 model lives on the diagonal, the model proposed in [12] lives at the point B.

For the metric $(\overline{3.4})$ the potential in $(\overline{3.2}),(\overline{3.3})$ takes the following form

$$
V(y)=p^{2}(1+\xi k|y|)^{2(\zeta / \xi-1)}+\frac{\left(\xi^{2}+15 \zeta^{2}\right) k^{2}}{4(1+\xi k|y|)^{2}}-(\xi+3 \zeta) k \delta(y) .
$$

We see that the Lorentz violation contributes the first term of the potential $V$, which is momentum-dependent. The ratio $\zeta / \xi$ determines the behavior of the potential at infinity. In our considerations we restrict ourselves to the case with "finite volume" of the fifth

\footnotetext{
${ }^{5}$ For instance, one could impose the constraint $\xi^{2}+\zeta^{2}=1$ and let $k$ play a role of curvature scale.
} 


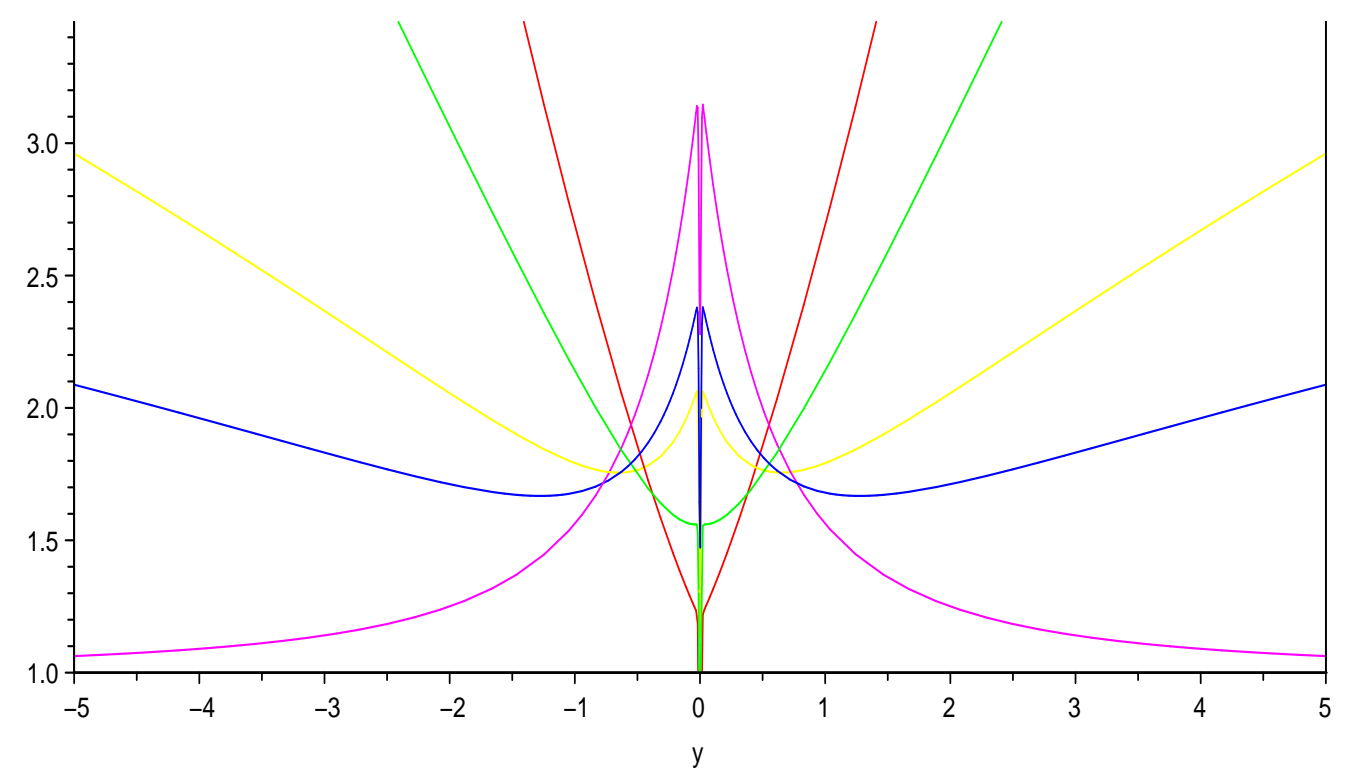

Figure 2: The effective potential for different values of $\zeta / \xi$. The curves are ordered by steepness: for the steepest one the ratio $\zeta / \xi$ is 0.3 , then the values $0.5,0.7,0.8,1$ follow. The brane is located at $y=0$ and $p=k=1$.

dimension $^{6}$ with the following condition satisfied

$$
\int_{-\infty}^{+\infty} d y \sqrt{g}<\infty,
$$

which in our parametrization means that $\xi+3 \zeta>0$. The corresponding domain in Fig. 1 has four regions I, II, III, and IV so let us briefly discuss the form of the potential for all these regions. First, we assume that both $\xi$ and $\zeta$ are nonnegative (regions I and II). If $\zeta / \xi>1$ then the first term in (3.5) increases at large $y$ thereby producing infinite walls to the potential $V$. Steepness of these walls is controlled by the same ratio $\zeta / \xi$. The case with $\xi=\zeta$ corresponds to the Lorentz invariant background of the RS2 model with $A d S_{5}$ as a bulk space. The case with $\zeta / \xi<1$ does not differ from the RS2 model qualitatively - the potential has the same kind of a shape. In the region III the potential (3.5) has poles at $|y|=-(\xi k)^{-1}$ but they are not physical, our coordinate system is not appropriate for this region. However, upon choosing a proper coordinates one can proceed with this region as well and observe similar behavior as in the region I. As far as we can conclude from (3.5), models located in the region IV do not qualitatively differ from models of type II, indeed, the potential has similar asymptotic behavior at infinity. At Fig. 2 we depicted the potential (3.5) at different positive values of the ratio $\zeta / \xi$.

Employing the considerations from the beginning of this section we can speculate on character of the perturbation spectra of type I and type II models. A full exact solution

\footnotetext{
${ }^{6}$ If the fifth dimension has an infinite volume then there is no in general well defined four-dimensional effective theory - the model appears to be five-dimensional without any localization properties on the brane. See some considerations in this directions e.g. at 14 .
} 


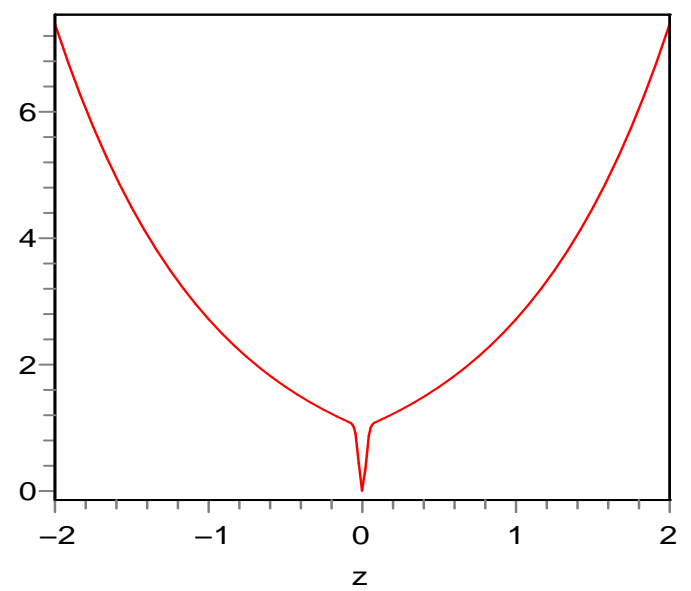

Figure 3: Shape of the model A potential. We put $p=k=1$ here.

for generic value of $\xi / \zeta$ is however not possible, nevertheless approximate analysis leads to desired understanding of the spectra of these models. Analogously with the considerations from the previous subsection we can straightforwardly state that the models of type I have a box-type potential and hence have discrete spectrum of the scalar perturbations, whereas the models of type II have decaying potential at infinity and plane wave excitations propagating along $y$-axis. The excitation spectrum of type II models is therefore continuous in general. Due to the delta-well it may contain a zero mode as well. Properties of type II world were discussed in [12] (point B). Now we shall investigate the scalar fluctuations in the completely different background A with almost opposite properties.

\subsection{The Model A. Generic Solution and Static Potential}

In order to proceed with the model A corresponding to the point $(0,1)$ in Fig. 1 one considers the Schrödinger equation in the form (3.1). The potential reads

$$
V(z)=p^{2} \mathrm{e}^{2 k|z|}+\frac{9}{4} k^{2}-3 k \delta(z) .
$$

We see that the potential tends to infinity as $|z| \rightarrow \infty$, thus we expect the perturbation spectrum to be discrete. Schematically the potential $(\overline{3.6})$ is shown in Fig. 3. First, we solve (3.1) with the potential $(3.6)$ for $z \neq 0$

$$
\chi^{\prime \prime}(z)+\left(E^{2}-p^{2} \mathrm{e}^{2 k|z|}-\frac{9}{4} k^{2}\right) \chi(z)=0
$$

then we impose the boundary conditions at the origin to take into account the delta-function term

$$
\left.\left[\chi^{\prime}(z)\right]\right|_{0}+3 k \chi(0)=0 .
$$

The normalizable solution is expressed via the following modified Bessel function

$$
\chi(z)=N \mathrm{~K}_{\nu}\left(\frac{p}{k} \mathrm{e}^{k|z|}\right),
$$


where $\nu=\sqrt{\frac{9}{4}-\frac{E^{2}}{k^{2}}}$ and $N$ is a normalization constant which is determined by the condition

$$
\int_{-\infty}^{+\infty} d z|\chi(z)|^{2}=1
$$

The matching condition $(3.7)$ at $z=0$ yields

$$
\frac{p}{k} \frac{\mathrm{K}_{\nu+1}\left(\frac{p}{k}\right)}{\mathrm{K}_{\nu}\left(\frac{p}{k}\right)}=\frac{3}{2}+\nu
$$

This equation relates the energy and momentum of modes, therefore it represents the dispersion relation. We consider it below.

Green Function. Let us now find the brane-to-bulk Green function for the scalar field $\phi$. In the momentum space it satisfies the following equation ${ }^{7}$

$$
\left[-\partial_{z}^{2}-E^{2}+p^{2} \mathrm{e}^{2 k|z|}+\frac{9}{4} k^{2}-3 k \delta(z)\right] \Delta_{p}(E, p, z)=\delta(z) .
$$

Using (3.8) one obtains the decaying solution to this equation outside of the brane

$$
\Delta_{p}(E, p, z)=C(E, p) \mathrm{K}_{\nu}\left(\frac{p}{k} \mathrm{e}^{k|z|}\right)
$$

where $C(E, p)$ is yet to be determined constant. Due to the presence of the delta-functions in $(3.11)$ one imposes the following constraint

$$
\left.\left[\partial_{z} \Delta_{p}(E, p, 0)\right]\right|_{0}+3 k \Delta_{p}(E, p, 0)=-1
$$

This equation determs the constant $C(E, p)$. Now we are ready to find the propagator. Let us put $z=0$ in order to extract the brane-to-brane propagator

$$
\Delta_{p}(E, p, 0)=\frac{2}{k}\left[\frac{p}{k} \frac{\mathrm{K}_{\nu+1}\left(\frac{p}{k}\right)}{\mathrm{K}_{\nu}\left(\frac{p}{k}\right)}-\nu-\frac{3}{2}\right]^{-1} .
$$

We see that the condition for the brane-to-brane propagator to have a pole exactly coincides with $(3.10)$. Later we show that $(3.10)$ has only real valued solutions in terms of $E$, hence all perturbation modes in our model are stable. In order to find the static brane-to-brane Green function we put $E=0$ and obtain it in the momentum space

$$
G_{p}(0) \equiv \Delta_{p}(0, p, 0)=\frac{1}{2 k}\left[\frac{p}{k} \frac{\mathrm{K}_{\frac{5}{2}}\left(\frac{p}{k}\right)}{\mathrm{K}_{\frac{3}{2}}\left(\frac{p}{k}\right)}-3\right]^{-1}=\frac{k}{2 p^{2}}+\frac{1}{2 p} .
$$

\footnotetext{
${ }^{7}$ Due to $S O(3)$ rotational brane symmetry the Green function depends only on absolute value of momentum $p$.
} 
Inverted Fourier transform leads us to the Green function in the coordinate representation which represents the static potential

$$
\begin{aligned}
G(r) & =\int \frac{d^{3} p}{(2 \pi)^{3}} G_{p}(0) \mathrm{e}^{i \mathbf{p r}}=\frac{4 \pi}{(2 \pi)^{3} r} \int_{0}^{+\infty} d p\left(\frac{k}{p}+1\right) \frac{\mathrm{e}^{i p r}-\mathrm{e}^{-i p r}}{2 i} \\
& =\frac{1}{2 \pi^{2} r}\left(k \int_{0}^{+\infty} d p \frac{\sin (p r)}{p}-2 i \int_{0}^{+\infty} d p \mathrm{e}^{i p r}\right)=\frac{k}{4 \pi r}\left(1+\frac{2}{\pi k r}\right) .
\end{aligned}
$$

The obtained potential describes the Coulomb (Newton) law with small correction at large distances compared with the curvature scale $1 / k$. At small distances the interaction becomes five-dimensional. Note that unlike the RS2 model [4] the correction to the potential has a different power: $r^{-2}$ instead of $r^{-3}$ for the RS2 model.

\subsection{Spectrum of the model A}

Let us now discuss the spectrum of the scalar sector in the model A. We proceed with expanding (3.10) at small and large momenta and derive dispersion relations for different excitation modes. The spectrum of the scalar field $\phi$ including the zero mode and higher modes is shown in Fig. 4. We observe that there exists zero mode and higher excitations. The behavior of the higher excitations at small momenta is of particular interest, as all curves collapse to one point $E=\frac{3}{2} k$ as $p \rightarrow 0$.

Zero mode at small momenta. From $(\overline{3.10})$ one observes that there exists a solution with $E(p) \rightarrow 0$ as $p \rightarrow 0$ which corresponds to the zero mode. The corresponding dispersion relation can be found if one performs an expansion in the vicinity of the point $E=0, p=0$, and after straightforward calculation one obtains

$$
E^{2}=3 p^{2}\left(1-\frac{p}{k}+\mathcal{O}\left(p^{2}\right)\right)
$$

We see that the zero mode dispersion relation is not Lorentz invariant. However, the low energy theory is approximately Lorentz invariant in the sense that the dispersion relation $E^{2} \approx 3 p^{2}$ has the form of the ordinary relativistic formula with rescaled speed of light. From (3.14) one finds the speed of propagation of scalar particles (group velocity $\partial E / \partial p$ ) which is equal to $\sqrt{3}$ and exceeds a speed of tightly bounded massless particles on the brane being equal to unity. It also exceeds the speed of scalar particles considered in [12] which is always less than 1. Therefore, one can expect that while moving in the parameter space in Fig. 1 from point $\mathrm{B}$ to point $\mathrm{A}$ throughout the upper right region, the speed of scalar particles will increase from the values found in $|12|$ to $\sqrt{3}$ derived here.

Let us calculate the zero mode contribution to the static brane Green function (3.12)

$$
G_{p}^{(0)}(0)=\frac{\left|\chi^{(0)}(0)\right|^{2}}{E^{2}}
$$




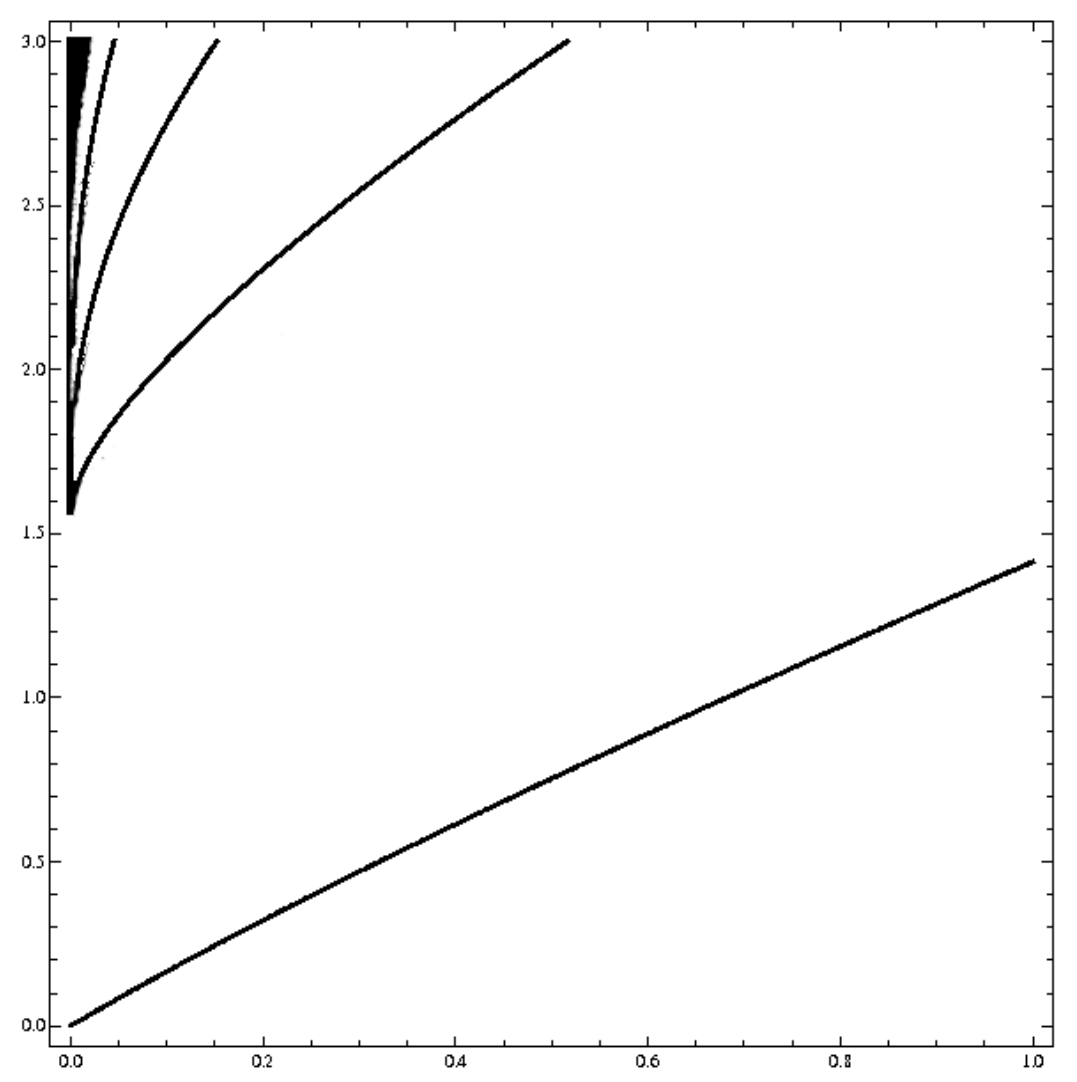

Figure 4: Spectrum of the field $\phi$. The energy $E$ in the vertical axis and the momentum $p$ in the horizontal axis are scaled in the units of $k$. The higher modes $n=1,2, \ldots$ start from $E=\frac{3}{2} k$. 
In order to find it one should know the zero mode normalization constant $N_{0}$ which can be determined at low momenta in the following way. One notes that $\partial \mathrm{K}_{\nu}(x) / \partial \nu=\mathrm{K}_{\nu}(x)(\log x+$ $\mathcal{O}(1))$ (see, e.g. $[15]$ ), so the zero mode wave function can be represented as

$$
\chi^{(0)}(z)=N_{0} \mathrm{~K}_{\frac{3}{2}}\left(\frac{p}{k} \mathrm{e}^{k|z|}\right)\left[1+\mathcal{O}\left(p^{2} \log \frac{p}{k} \mathrm{e}^{k|z|}\right)\right] .
$$

After that the normalization constant can be calculated from (3.9)

$$
N_{0}^{2}=k \frac{3}{\pi}\left(\frac{k}{p}\right)^{3}\left[1+\mathcal{O}\left(\frac{p^{2}}{k^{2}} \log \frac{p}{k}\right)\right] .
$$

Given $N_{0}$ and substituting the zero mode dispersion relation $(3.14)$ together with $(3.16)$ into (3.15) one finds the zero mode contribution to the static Green function

$$
G_{p}^{(0)}(0)=\frac{k}{2 p^{2}}+\frac{1}{2 p}+\mathcal{O}(\log p) .
$$

We see that at low momenta the Green function (3.13) is saturated by the zero mode. The corrections $\mathcal{O}(\log p)$ emerging in (3.17) should be canceled out by contributions from higher modes.

Higher modes at small momenta. As was mentioned above the mode equation (3.1) can be written as a Schrödinger equation with a potential dependent on a particle momentum. We can apply WKB method here for understanding the spectrum of higher excitations 8 . The quantization law reads 9

$$
\oint \sqrt{E_{n}^{2}-V(z)} d z=2 \pi n
$$

where the potential $V(z)$ is given in $(3.6)$. Because of $\mathbb{Z}_{2}$ reflection symmetry of the potential we have

$$
2 \cdot 2 \int_{0}^{z_{n}} \sqrt{E_{n}^{2}-V(z)} d z=2 \pi n
$$

where the classical turning points have the following coordinates for each $n$

$$
z_{n}= \pm \frac{1}{2 k} \log \frac{E_{n}^{2}-\frac{9}{4} k^{2}}{p^{2}} .
$$

Calculating integral in the l.h.s. of $(3.19)$ one has

$$
-\sqrt{E_{n}^{2}-\frac{9}{4} k^{2}-p^{2}}+\sqrt{E_{n}^{2}-\frac{9}{4} k^{2}} \operatorname{Arctanh} \sqrt{1-\frac{p^{2}}{E_{n}^{2}-\frac{9}{4} k^{2}}}=\frac{\pi k}{2} n .
$$

\footnotetext{
${ }^{8}$ In order to find the dispersion relation for the higher modes one can solve $(3.10)$ at the low momenta directly. However, we do not perform this analysis here. For the fermionic sector, where the spectrum of higher modes will appear to be very much similar to the scalar one discussed here, we shall expand an analog of $(3.10)$ directly.

${ }^{\bar{y}}$ In our calculations we ignore $\delta$-function contribution to the potential $V$ as it brings only small correction to the integral in the l.h.s of the WKB formula. Therefore we can also neglect $\frac{1}{2}$ in the r.h.s. as it is irrelevant for our precision.
} 
Expansion for $0<p \ll k$ yields

$$
-\sqrt{E_{n}^{2}-\frac{9}{4} k^{2}} \log \frac{p}{k}=\frac{\pi k}{2} n
$$

or

$$
E_{n}^{2}=\frac{9}{4} k^{2}+\frac{\pi^{2} k^{2} n^{2}}{4 \log ^{2} \frac{p}{k}} .
$$

One can show that the WKB method used here is applicable provided that $p / k<\mathrm{e}^{-n}$.

The obtained result $(3.20)$ becomes more transparent if one notes that at small momenta the potential (3.6) can be approximated by the box-type potential

$$
p^{2} \mathrm{e}^{2 k|z|} \simeq\left[\begin{array}{cl}
0 & \text { if }|z|<-\frac{1}{k} \log \frac{p}{k} \\
\infty & \text { if }|z|>-\frac{1}{k} \log \frac{p}{k}
\end{array} .\right.
$$

Then the dispersion relation (3.20) immediately follows. Note also, that if $p=0$ the spectrum becomes continuous what corresponds to a particle which can propagate to infinity in complete agreement with the results of Sec. 2 .

Large momenta. At large momenta $p \gg k$ one can neglect the factor of $\frac{3}{2}$ in the r.h.s. of $(3.10)$ and $(3.10)$ becomes equivalent to the following condition

$$
\mathrm{K}_{\nu}^{\prime}\left(\frac{p}{k}\right)=0
$$

This condition is considered in App. $\mathrm{A}$ in the context of fermionic spectrum at large momenta (see $(\overline{A .8})$ ). The result is

$$
E=p+\mathcal{O}\left(p^{\frac{1}{3}}\right) .
$$

Thus at large momenta, as expected, the theory becomes Lorentz invariant.

\section{Fermionic Fluctuations}

In this section we discuss the spectrum of fermionic fluctuations in braneworlds with broken Lorentz invariance. One can perform a qualitative analysis for generic $(\xi, \zeta)$ configuration (3.4) and reduce the appropriate five dimensional Dirac equations to a Schrödinger-type equations. Then, analogously to the scalar sector discussed at Sec. 3, one can establish that the asymptotic behavior of the metric at infinity is responsible for the actual pattern of the spectrum. For instance, if $\xi \geq \zeta$ the spectrum is continuous. In this section we refrain from considering qualitative picture with generic ansatz (3.4) but focus our attention on the model A where exact solution is obtained and analyzed.

We consider the four-component spinor field $\Psi$ on the five-dimensional manifold with metric $(1.3)$

$$
S=\int d t d \mathbf{x} \int_{-\infty}^{+\infty} d z \sqrt{g}\left(i \bar{\Psi} \not \nabla \Psi-m_{\psi} \bar{\Psi} \Psi\right)
$$


where $m_{\psi}$ is the five-dimensional mass of the fermion which may however depend on $z$. In this section we study two cases:

i) $m_{\psi}=m \cdot \operatorname{sign}(z)$. It corresponds to a usual (Lorentz invariant) way of localization of fermions in extra dimensional setups (see, e.g. $[16 \mid$ and for more recent treatments [17|). It is worth noting that in this case the action (4.1) is invariant under $\mathbb{Z}_{2}$ reflection symmetry $z \rightarrow-z$ if the fermion field transforms as

$$
\Psi(-z)=\mp \gamma_{5} \Psi(z)
$$

where phase $\mp$ can be only determined by fermion interactions.

ii) $m_{\psi}=m$. In this case we deal with a usual massive fermion and the action does not possess the $\mathbb{Z}_{2}$ symmetry. It does not play a role, however, since this case has only an illustrative character and we are interested in the limit $m \rightarrow 0$ which can be easily obtained in this case and which restores $\mathbb{Z}_{2}$ symmetry.

Generic Solution. The Dirac equation obtained from (4.1) reads

$$
i \Gamma^{A} \nabla_{A} \Psi(x, z)-m_{\psi} \Psi(x, z)=0,
$$

where $\nabla_{A}=\partial_{A}+\omega_{A}$ is the covariant derivative with the spin-connection

$$
\omega_{A}=\left(0,-\frac{i}{2} k \gamma_{5} \gamma_{i} \mathrm{e}^{-k|z|}, 0\right) \operatorname{sign}(z) .
$$

The five matrices $\Gamma^{A}(A=0,1,2,3,5)$ obeying the Clifford algebra relations

$$
\left\{\Gamma^{A}, \Gamma^{B}\right\}=2 g^{A B},
$$

are related to the $\gamma$-matrices $(\mu, \nu=0,1,2,3)$

$$
\left\{\gamma^{\mu}, \gamma^{\nu}\right\}=2 \eta^{\mu \nu}, \quad \gamma^{5}=\gamma_{5}=i \gamma^{0} \gamma^{1} \gamma^{2} \gamma^{3}
$$

as follows

$$
\Gamma^{0}=\gamma^{0}, \quad \Gamma^{i}=\mathrm{e}^{k|z|} \gamma^{i}, \quad \Gamma^{5}=-\Gamma_{5}=-i \gamma^{5} .
$$

In order to simplify further calculations it is convenient to work with the rescaled field

$$
\Psi=\mathrm{e}^{\frac{3}{2} k|z|} \psi
$$

and apply the four-dimensional Fourier transform to the field $\psi$

$$
\psi(t, \mathbf{x}, z)=\frac{1}{(2 \pi)^{2}} \int d E d \mathbf{p} \mathrm{e}^{i E t-i \mathbf{p x}} \psi(E, \mathbf{p}, z)+\text { h.c. } .
$$

In this relation and in what follows we assume that $E>0$. Then due to (4.5) the normalization condition for the field $\psi(E, \mathbf{p}, z)$ has the canonical form

$$
\int_{-\infty}^{+\infty} d z \sqrt{g} \bar{\Psi} \gamma_{0} \Psi=\int_{-\infty}^{+\infty} d z \psi^{\dagger}(E, \mathbf{p}, z) \psi(E, \mathbf{p}, z)=1 .
$$


In terms of the new field the Dirac equation (4.3) takes the form

$$
\left(E \gamma^{0}-\mathrm{e}^{k|z|} \gamma^{3} p-\gamma^{5} \partial_{z}\right) \psi+m_{\psi} \psi=0 .
$$

where we set $\mathbf{p}=(0,0, p)$ for simplicity. In order to solve this equation we use the following decomposition of the spinor

$$
\psi=\sum_{\alpha, \beta} \phi_{\alpha, \beta}(E, p, z) U_{\alpha, \beta}
$$

where $\alpha$ and $\beta$ independently take values,$\pm \phi_{\alpha, \beta}$ are four unknown functions, and $U_{\alpha, \beta}$ are four constant spinors with the following properties

$$
\begin{aligned}
2 S_{3} U_{\alpha, \beta} & \equiv i \gamma_{1} \gamma_{2} U_{\alpha, \beta}=\alpha U_{\alpha, \beta} \\
\gamma_{0} U_{\alpha, \beta} & =\alpha \beta U_{\alpha, \beta} \\
\gamma_{3} U_{\alpha, \beta} & =\beta U_{\alpha,-\beta} \\
\gamma_{5} U_{\alpha, \beta} & =-U_{\alpha,-\beta} .
\end{aligned}
$$

Let us say a few words concerning the above relations. Because of $S_{3}$ (the third component of the spin) commutes with (4.7) it is easy to use the basis composed of the eigenvectors of $S_{3}$ - this is what the first property tells us. Since $\gamma_{0}$ commutes with $S_{3}$ one can choose the basis in which the eigenvectors of $S_{3}$ are eigenvectors of $\gamma_{0}$ as well - this is what the second property tells us. The first two properties correspond to fixing of the orthogonal basis up to four unknown constants. Since $\gamma_{3}$ commutes with $S_{3}$ and anticommutes with $\gamma_{0}$ one can check that $\gamma_{3} U_{\alpha, \beta} \sim U_{\alpha,-\beta}$ - the third property fixes the constant in this relation and, thereby, fixes the basis up to two constants which can be found (up to phases) from the normalization conditions

$$
U_{\alpha^{\prime}, \beta^{\prime}}^{\dagger} U_{\alpha, \beta}=2 \delta_{\alpha^{\prime} \alpha} \delta_{\beta^{\prime} \beta} .
$$

The last property follows directly from the first three properties and from the definition of $\gamma_{5}(4.4)$

Substituting the expansion (4.8) into (4.7) one obtains the following equation

$$
\left(\partial_{z}-\beta p \mathrm{e}^{k|z|}\right) \phi_{\alpha, \beta}=\left(\alpha \beta E-m_{\psi}\right) \phi_{\alpha,-\beta} .
$$

Acting on both sides of this equation by the operator $\partial_{z}+\beta p \mathrm{e}^{k z}$ and using $(4.10)$ with $\beta \rightarrow-\beta$ one ends up with the second order equation

$$
\left(\partial_{z}^{2}+E^{2}-m^{2}-p^{2} \mathrm{e}^{2 k|z|}-\beta p k \operatorname{sign}(z) \mathrm{e}^{k|z|}\right) \phi_{\alpha, \beta}=-2 m \delta(z) \phi_{\alpha,-\beta}
$$

where we assume that $m_{\psi}=m \cdot \operatorname{sign}(z)$. It is easy to check that the normalizable solution of $(4.11)$ at $z>0, p>0$ has the form ${ }^{10}$

$$
\phi_{\alpha, \beta}^{>}(z)=C_{\alpha, \beta}^{>} \xi_{\beta}\left(\frac{p}{k} \mathrm{e}^{k|z|}\right),
$$

where

$$
\xi_{\beta}(x)=\sqrt{x}\left[\mathrm{~K}_{\nu+\frac{1}{2}}(x)-\beta \mathrm{K}_{\nu-\frac{1}{2}}(x)\right] .
$$

\footnotetext{
${ }^{10}$ No summation over repetitive indexes $\alpha$ and $\beta$ is assumed.
} 
In what follows we use the superscripts " $>$ " for the functions at $z>0$ and " $<$ " for the functions at $z<0, C_{\alpha, \beta}^{>,<}$are constants, and $\nu=k^{-1} \sqrt{m^{2}-E^{2}}$. It is worth noting that the Dirac operator in the problem under consideration is Hermitian. Thus the energy $E$ has to be real (in fact it follows directly from the dispersion relation). It means in particular that $\nu$ has to be either real $(E<m)$ or pure imaginary $(E>m)$. In both cases the sign of $\nu$ does not play any role: it can be absorbed into the definitions of $C_{\alpha, \beta}$ because of $\xi_{\beta}(-\nu)=-\beta \xi_{\beta}(\nu)$. So we assume that either real $(E<m)$ or imaginary $(E>m)$ part of $\nu$ is positive.

One can see from (4.11) that under the reflection transformation $z \rightarrow-z$ only the last term in the 1.h.s. changes the sign. Thus the solution at $z<0$ can be represented as

$$
\phi_{\alpha, \beta}^{<}(z)=C_{\alpha, \beta}^{<} \xi_{-\beta}\left(\frac{p}{k} \mathrm{e}^{k|z|}\right) .
$$

It follows from (4.11) that the solutions for $p<0$ can be obtained from $(4.12)$ and $(4.13)$ by interchanging $\beta \rightarrow-\beta$. The eight unknown constants $C_{\alpha, \beta}^{>,<}$have to be determined from the normalization condition $(\overline{4.6})$, from the continuity conditions at the origin

$$
\phi_{\alpha, \beta}^{>}(0)=\phi_{\alpha, \beta}^{<}(0)
$$

and from the matching conditions for the derivatives which follow from $(4.11)$

$$
\partial_{z} \phi_{\alpha, \beta}^{>}(0)-\partial_{z} \phi_{\alpha, \beta}^{<}(0)=-2 m \phi_{\alpha,-\beta}(0)
$$

At fixed $\alpha,(4.14)$ and $(4.15)$ form a set of four linear homogeneous equations for the four unknown constants $C_{\alpha, \beta}^{>,<}$. Hence in order this set to be self-consistent its determinant should be equal to zero. Calculating the determinant we end up with the equation

$$
t^{2}\left(\frac{\partial}{\partial t}\left(\xi_{+}(t) \xi_{-}(t)\right)\right)^{2}=\frac{4 m^{2}}{k^{2}}\left(\xi_{+} \xi_{-}\right)^{2},
$$

where $t=p / k$. This equation represents the dispersion relation $E=E(p)$ for the fermion field in question.

The equations $(4.15)$ and $(4.16)$ can be considerably simplified if one notes that the functions $\xi_{\beta}$ satisfy the relations

$$
\frac{d \xi_{\beta}(x)}{d x}=-\frac{\nu}{x} \xi_{-\beta}+\beta \xi_{\beta}
$$

Using these identities one obtains from $(4.14)$ and $(4.15)$ the relations for the constants $C_{\alpha, \beta}^{>,<}$

$$
\begin{aligned}
C_{\alpha, \beta}^{<} & =\frac{\xi_{\beta}(t)}{\xi_{-\beta}(t)} C_{\alpha, \beta}^{>}, \\
C_{\alpha,-\beta}^{>} & =\gamma \frac{\xi_{\beta}(t)}{\xi_{-\beta}(t)} C_{\alpha, \beta}^{>},
\end{aligned}
$$

where $\gamma= \pm 1$ corresponds to the two possible signs of the square root of (4.16). Making use of $(4.9)$ one verifies that $\gamma$ corresponds to the $\mp$ signs in $(4.2)$. Thus $\gamma$ remains ambiguous 
and can be only fixed by the fermion interactions. As it follows from (4.17), at fixed $\alpha$ we have only one unknown constant, say

$$
C_{\alpha} \equiv \xi_{+}(t) C_{\alpha,+}^{>}=\gamma \xi_{-}(t) C_{\alpha,-}^{>}
$$

which is fixed by the normalization condition (4.6). It means that at fixed $\alpha$ and positive energy we have only one degree of freedom - a particle with the polarization $\alpha$.

It is worth noting here that $\nu$ cannot vanish at any value of energy. Indeed, if $\nu=0$ then $\xi_{+} \equiv 0$. It means that $C_{\alpha,-} \equiv 0$ (see $(4.17)$ ), and, so, both $\phi_{\alpha, \pm} \equiv 0$, i.e. there is no normalized solution in this case.

Let us now substitute $(4.17)$ into $(4.12)$ and afterwards into $(4.10)$. Then one finds that

$$
\frac{\xi_{-\beta}(t)}{\xi_{\beta}(t)}=\gamma \frac{\mu-\alpha \beta \varepsilon}{\nu}
$$

where we have introduced the dimensionless energy $\varepsilon=E / k$ and mass $\mu=m / k$. In fact this equation does not depend on $\beta$ : if one changes $\beta \rightarrow-\beta$ then by making use of the definition of $\nu$ in the r.h.s of $(4.19)$ one obtains the same equation. Thus $(4.19)$ is equivalent to the equation

$$
\frac{\xi_{-}(t)}{\xi_{+}(t)}=\gamma \frac{\mu-\alpha \varepsilon}{\nu}
$$

This relation is nothing but the dispersion relation $E=E(p)$. One can check that $(4.20)$ follows directly from (4.16). However, from (4.16) one can obtain another branch of solutions which corresponds to the substitution $\alpha \rightarrow-\alpha$ in (4.20). This branch does not satisfy (4.10) and should be rejected.

Let us now consider the case $m_{\psi}=m$. In this case the term with the delta-function in the r.h.s. of $(\overline{4.11})$ is absent. Equations $(4.12)-(4.14)$ still hold, in $(4.15),(4.16)$ the r.h.s. vanishes. Thus the equation yielding the dispersion relation in this case (an analog of $(4.20)$ ) follows from $(4.16)$

$$
\frac{\xi_{-}(t)}{\xi_{+}(t)}=i \gamma,
$$

where $\gamma= \pm 1$. An analog of $(4.17)$ reads as follows

$$
\begin{aligned}
C_{\alpha, \beta}^{<} & =\frac{\xi_{\beta}(t)}{\xi_{-\beta}(t)} C_{\alpha, \beta}^{>}=-i \beta \gamma C_{\alpha, \beta}^{>}, \\
C_{\alpha,-\beta}^{>} & =\frac{\nu}{\mu-\alpha \beta \varepsilon} C_{\alpha, \beta}^{>} .
\end{aligned}
$$

The second equation in (4.22) follows from (4.10). This equation in fact does not depend on $\beta$ - interchanging $\beta \rightarrow-\beta$ leads to the same equation if one takes into account the definition of $\nu$. Thus we have no other constraints on the constants $C_{\alpha, \beta}$ and at fixed $\alpha$ we have only one degree of freedom.

Let us now discuss the spectrum of the fields $\phi_{\alpha, \beta}$ which follows from $(\overline{4.20})$ and $(4.21)$. 


\section{Spectrum of the model A}

Here we give a summary of the fermionic spectrum of the model A at low and high momenta and at different values of the bulk mass. The detailed calculations are given in App. A. The spectrum pattern at low momenta is shown in Fig. 5 . We begin with the case $m_{\psi}=m \cdot \operatorname{sign}(z)$.

Zero mode at low momenta. A zero or gapless mode is a state with energy $E(p) \rightarrow 0$ as momentum $p \rightarrow 0$. From the four-dimensional point of view a zero mode corresponds to a massless state. As we will see the zero mode is present in the fermionic spectrum of the model both in the case $m=0$ and $m \neq 0$. However, the dependence of the energy on the momentum $p \ll m, k$ is different for different values of the bulk mass $m$. Here we consider only the case of nonzero mass. As is shown in App. A, the dispersion relation has the following form

$$
E=\left(\frac{2 m}{2 m-k}\right) p+2 m \frac{\Gamma\left(\frac{1}{2}-\frac{m}{k}\right)}{\Gamma\left(\frac{1}{2}+\frac{m}{k}\right)}\left(\frac{p}{2 k}\right)^{\frac{2 m}{k}}+\mathcal{O}\left(p \log \frac{p}{k}\right)
$$

From this formula the following three regimes can be derived

- At $m>k / 2$ the dispersion relation is

$$
E \simeq\left(\frac{2 m}{2 m-k}\right) p
$$

We see that in the limit $m \gg k$ the dispersion relation becomes Lorentz invariant as it should be as this limit corresponds to the flat case.

- At $m=k / 2$ we have

$$
E \simeq-p \log \frac{p \mathrm{e}^{\gamma_{E}}}{2 k}
$$

where $\gamma_{E} \simeq 0.577$ is Euler constant.

- At $0<m<k / 2$ we have

$$
E \simeq 2 m \frac{\Gamma\left(\frac{1}{2}-\frac{m}{k}\right)}{\Gamma\left(\frac{1}{2}+\frac{m}{k}\right)}\left(\frac{p}{2 k}\right)^{\frac{2 m}{k}}
$$

In all these regimes it turns out that $\alpha=-1$, and $\gamma=1$. Thus the zero mode describes a massless polarized particle with helicity -1 .

Higher modes at low momenta. We start with the case $m_{\psi}=m \cdot \operatorname{sign}(z)$. Similarly to the scalar spectrum discussed in Sec. 3 one can study the spectrum of higher fermionic modes employing the WKB approximation. Here we, however, present the dispersion relations for 

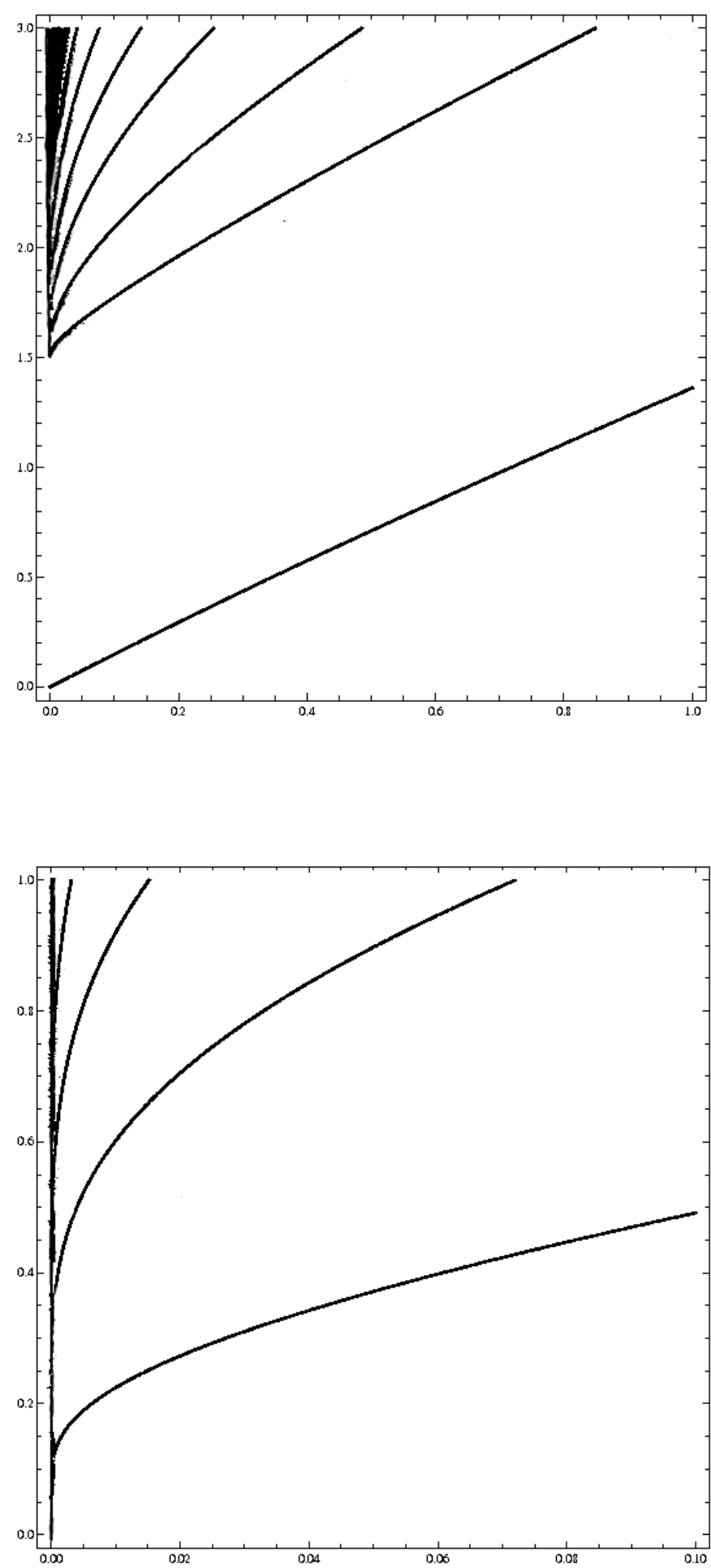

Figure 5: Fermionic spectra $\varepsilon=\varepsilon(t)$ for $\mu=\frac{3}{2}, \gamma=1$ and $m_{\psi}=m \cdot \operatorname{sign}(z)$ (upper plot), and $\mu=0$ (lower plot). Straight line starting from the origin of the upper plot shows the zero mode dispersion relation. Other curves originating from the point $\left(0, \frac{3}{2}\right)$ show dispersion laws for higher modes at different $n=1,2, \ldots$. At the lower plot all curves start from the origin. 
the higher modes obtained directly from $(4.20)$. Solving this equation at $p \ll m, k$ one finds (the detailed calculations are given in App. A)

$$
E_{n}=m \sqrt{1+\left(\frac{\pi n k}{2 \Psi\left(\frac{1}{2}\right) m-\gamma k-2 m \log \frac{p}{2 k}}\right)^{2}} \simeq m \sqrt{1+\left(\frac{\pi n k}{2 m \log \frac{p}{k}}\right)^{2}}
$$

where $\Psi(z)$ is Euler digamma function and $n \in \mathbb{N}$ is the mode number. The helicity depends on the mode number as $\alpha=(-1)^{n+1}$. The latter approximate equality in $(4.27)$ is obtained by the WKB approximation and is similar to (3.20) for the scalar case. It is worth noting that the first equality in (4.27) is valid only for $n$ such that the term in the parenthesis is less than unity. At $n$ large enough the character of the spectrum does not change: it remains to be discrete and $E \rightarrow m$ as $p \rightarrow 0$.

It follows from (4.27) that at fixed level number $n$ there are two states with different energies $^{11} E(\gamma=1)$ and $E(\gamma=-1)$. Moreover, these two massive states are polarized and have the same helicity $\alpha=(-1)^{n+1}$. So the spectrum of the model for the positive energies is completely non-degenerate.

Now we consider the case with the constant bulk mass $m_{\psi}=m$ at small momentum $p \ll m, k$. In this case we obtain the following dispersion relation

$$
E_{n}=\sqrt{m^{2}+\left(\frac{k \pi(2 n+1)}{4 \Psi\left(\frac{1}{2}\right)-4 \log \frac{p}{2 k}}\right)^{2}}
$$

where $n$ is a nonnegative integer number. We also have $\gamma=(-1)^{n+1}$. It is worth noting that $\gamma$ rather then $\alpha$ is fixed in this case and $E_{n}$ does not depend on $\alpha$ at all. Thus for fixed $n$ the spectrum is doubly degenerate - there are two modes with helicities $\alpha= \pm 1$. One also sees that there is a smooth limit $m \rightarrow 0$ in this case in which all modes become gapless.

Modes at high momenta. At momenta large enough, $p \gg m, k$, the dispersion relations for all modes in the both cases $m_{\psi}=m \cdot \operatorname{sign}(z)$ and $m_{\psi}=m$ become

$$
E=p+\mathcal{O}\left(p^{\frac{1}{3}}\right)
$$

and the correction in $(4.29)$ is positive. Thus at large momenta the Lorentz invariant dispersion relation is restored.

Chiral properties of solutions. It is a standard lore that in the Lorentz invariant case for $m_{\psi}=m \cdot \operatorname{sign}(z)$ a zero mode appears in the spectrum and this zero mode is chiral from the four-dimensional point of view. Let us consider what happens in our case. Using (4.9) one finds

$$
\psi_{L, R}=\frac{1 \mp \gamma_{5}}{2} \psi=\frac{1}{2} \sum_{\alpha, \beta} U_{\alpha, \beta}\left(\phi_{\alpha, \beta} \pm \phi_{\alpha,-\beta}\right) .
$$

\footnotetext{
${ }^{11}$ It worth to note that $\gamma(\operatorname{sign}$ in $(4.2))$ plays a role of the parameter of the model. Thus for the fixed $\gamma$ there is only one state.
} 
From this equation by means of $(4.12)$ and $(4.18)$ one has

$$
\psi_{L, R}^{>}=\left(\frac{\xi_{+}\left(t \mathrm{e}^{k|z|}\right)}{\xi_{+}(t)} \pm \gamma \frac{\xi_{-}\left(t \mathrm{e}^{k|z|}\right)}{\xi_{-}(t)}\right) \frac{1}{2} \sum_{\alpha} C_{\alpha}\left(U_{\alpha,+} \pm U_{\alpha,-}\right) .
$$

The corresponding expression at $z<0$ can be obtained from $(4.30)$ by interchanging $\xi_{-} \leftrightarrow \xi_{+}$. Thus both left-handed and right-handed components do not vanish as functions of $z$. In particular, the zero mode contains both left-handed and right-handed spinors. However, for the zero mode $\gamma=1$ and the right-handed spinor vanishes on the brane $z=0$. So, in this respect, one can say that only left-handed spinor is localized on the brane. This statement is true for higher modes as well: if $\gamma=1$ only left-handed spinors are localized on the brane, whereas, right-handed spinors are localized for $\gamma=-1$. In both cases depending on $\alpha$ the modes have different helicities (for the zero mode $\alpha=-1$ and helicity is -1 ). In Fig. 6 some mode profiles from $(4.30)$ are shown.
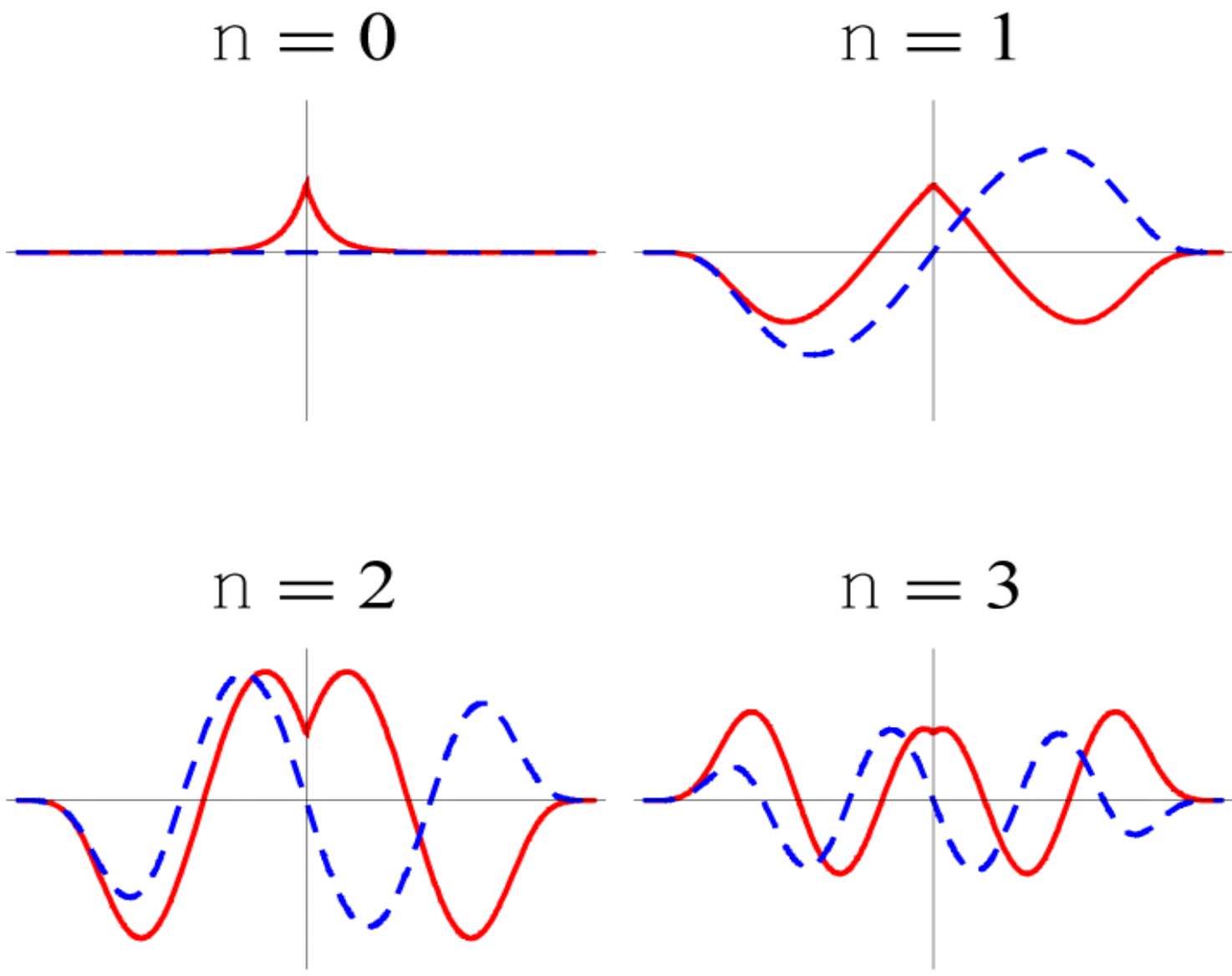

Figure 6: Mode profiles at $n=0,1,2,3$ for $m=2 k, p=0.01$. Red solid curves correspond to left-handed and dashed blue curves correspond to right-handed spinors. On the upper left plot $(\mathrm{n}=0)$ the right-handed component is strongly suppressed. 
In the case $m_{\psi}=m$ an analog of $(4.30)$ is

$$
\psi_{L, R}^{>}=\sum_{\alpha}\left(\frac{\xi_{+}\left(t \mathrm{e}^{k|z|}\right)}{\xi_{+}(t)} \pm i \gamma \frac{\nu}{\mu-\alpha \varepsilon} \frac{\xi_{-}\left(t \mathrm{e}^{k|z|}\right)}{\xi_{-}(t)}\right) \frac{C_{\alpha}}{2}\left[U_{\alpha,+} \pm U_{\alpha,-}\right]
$$

so the chiral components do not vanish even on the brane. However, in the massless limit the expression in the parentheses in $(4.31)$ tends to

$$
\frac{\xi_{+}\left(t \mathrm{e}^{k|z|}\right)}{\xi_{+}(t)} \pm \gamma \alpha \frac{\xi_{-}\left(t \mathrm{e}^{k|z|}\right)}{\xi_{-}(t)}
$$

Thus depending on $\gamma \alpha$ either left-handed $(\gamma \alpha=1)$ or right-handed $(\gamma \alpha=-1)$ modes are localized on the brane (again in the sense that the corresponding spinors vanish on the brane). In particular, for the lowest modes $(n=0) \gamma=-1$ the localized spinor is left-handed if helicity is $\alpha=-1$ and right-handed in the opposite case. Making an insight into (4.31) we see that even for the constant mass localization of fermionic perturbations is possible. The reason for that is based on the special geometry we work in and on negative curvature of the space [18].

Propagation velocity and relation to scalars. To conclude this section let us note that from eqs. $(4.24)-(\overline{4.29})$ one sees that group velocity $\partial E / \partial p$ in all regimes is greater than unity. Moreover, it follows from eqs. (4.25), (4.26) and (4.27), the group velocity tends to infinity as $p \rightarrow 0$. Thus in order to have equal velocities at low momenta for the scalar $\left(c_{s}=\sqrt{3}\right)(3.14)$ and the fermionic zero mode one needs to involve some sort of fine tuning. Namely, one assumes that $m>k / 2$ (only in this case the group velocity is finite), uses (4.24), and solves the equation

$$
\sqrt{3}=\frac{2 m}{2 m-k}
$$

The solution is $\frac{m}{k} \simeq 1.18>1 / 2$ which confirms that our assumption is valid.

\section{Conclusions}

In this work we investigated perturbation spectra of the scalar and the spin- $\frac{1}{2}$ fermion fields in the extra dimensional setups with broken Lorentz invariance in the bulk solutions found in [1]. In the beginning we qualitatively discussed the relation between asymptotic behavior of the bulk metric tensor at infinity in an extra dimension and localization of higher (nonzero) modes by analyzing geodesic equation in the given metric. Then for the massless scalar field in the bulk we performed the spectrum analysis by reducing the corresponding mode equation to the Schrödinger equation with a potential dependent on the metric and particle momentum. Existence of a zero mode is given rise by the presence of a deltawell in a potential. We have constructed the parameter space of the bulk metrics used in [1] with explicitly broken Lorentz invariance in the bulk and discussed how spectra of excitations behave in different domains of this space. It has been shown that if the $g_{00}$ metric coefficient increases faster then $g_{i j}$ as the fifth coordinate tends to infinity, then higher modes of field fluctuations are not localized and the spectrum is continuous; if the 
opposite case is realized then a spectrum is discrete and all modes are localized on the brane. Localization properties may, however, depend on three-dimensional momentum. In the end of Sec. 3 we have elaborated on the model with $g_{00}=1$ and $g_{i j}=\mathrm{e}^{-2 k|z|} \delta_{i j}$ as contrasted to the model [12], in which $g_{00}$ is warped while $g_{i j}$ is not.

The static potential for the massless scalar field was derived. Unlike the RS2 case our potential acquires different power correction at small distances which could make the two models experimentally distinguishable.

The dispersion relation for the zero mode was obtained and appeared to break Lorentz invariance explicitly. The speed of scalar massless particles propagating along the brane (which is governed by the zero mode dispersion relation) was found to be larger then the speed of tightly bound massless particle on the brane. Recall that in $[12]$ the speed was smaller and one can expect, that for intermediate values of Lorentz violating parameters a speed of scalar particles will be bounded by our result from above and by the result $|12|$ from below. However, for completeness, other domains of the parameter space Fig. 1 have to be analyzed.

In Sec. 4 spinor fluctuations were investigated on the example of the model A. We have explicitly found the solution of the Dirac equation with the mass term $m_{\psi}=m \cdot \operatorname{sign}(z)$ and derived the corresponding dispersion relations. We found fermionic spectra for different values of the ratio $m / k$. Generically these spectra appeared to be almost the same as in the scalar case except for the zero mode dispersion relation. For $m>\frac{1}{2} k$ the energy $E$ is proportional to the momentum $p$ with the coefficient dependent on this ratio; for $m=\frac{1}{2} k$ we had $E \sim p \log p$; and $m / k$-dependent power law behavior for $m<\frac{1}{2} k$. Moreover, it turns out that all modes including the zero mode are polarized but nonchira] ${ }^{12}$ unlike the usual Lorentz invariant case where an appeared zero mode is always chiral. Thus the energy spectrum is non-degenerate. From the four-dimensional point of view the nonchiral character of the zero mode means that it is not described by the Weyl equation - there is a mixing between left-handed and right-handed spinors (mass-like term) which depends on three-dimensional momentum and disappears in the limit of small momenta. Apart from that, chirality is precisely restored on the brane since either left or right-handed components of spinor wave functions vanish on the brane (the same is true for the higher modes as well).

We have also investigated the case $m_{\psi}=m$. It has an illustrative character due to the broken $\mathbb{Z}_{2}$ bulk symmetry. One can take the limit $m \rightarrow 0$ and $\mathbb{Z}_{2}$ symmetry is restored. Unlike all the other known braneworld models, a zero mode does exist even for $m=0$ which can be considered as a consequence of our special geometry and negative curvature of the space [18]. Namely, all excitation modes can be treated as zero modes since for all modes energy vanishes as $p \rightarrow 0$, unlike the scalar massless case where there is a gap $3 k / 2$ between zero and higher modes. However, the modes are not polarized and the energy levels are doubly degenerate. Moreover, all modes are nonchiral as in the $m_{\psi}=m \cdot \operatorname{sign}(z)$ case.

Both in the scalar and fermion cases we found that the spectra of higher modes have very peculiar properties - at small momenta energies of all modes tend to one and the same value independent of the mode number. From the four-dimensional point of view it means that all excitations have the same mass. However, on the other hand a particle with precisely zero momentum becomes delocalized and may freely escape from the brane. It is

\footnotetext{
${ }^{12}$ The modes correspond to a mixture of left-handed and right-handed spinors with certain coefficients.
} 
worth stressing that for a particle somehow localized in the three-dimensional space (wave packet) the momentum cannot be exactly equal to zero due to the uncertainty principle.

To conclude, the considered model possesses a lot of unusual and remarkable properties which are interesting from theoretical as well as from phenomenological points of view.

Note added. Recently we found an interesting paper $[19]$ where the authors deal with metric deformations which break Lorentz invariance similar to those considered in our work. In [20] an explicit comparison of the two solutions is made. The work [19] represents a prospective programme of gauge/gravity correspondence for theories with broken Lorentz invariance.

\section{Acknowledgements}

The authors are grateful to S. Dubovsky, D. Gorbunov, J. Kinney, D. Levkov, S. Sibiryakov, F. Spill, A. Zayakin for fruitful discussions and especially to V. Rubakov and A. Vainshtein for reviewing the final version of the manuscript. M.L. would like to thank Service de Physique Théorique, Université Libre de Bruxelles where part of this work was done under support in part by the Belspo:IAP-VI/11 and IISN grants, for kind hospitality. P.K. thanks MaxPlanck-Institut für Gravitationsphysik (Albert-Einstein-Institut) where part of his work was done for support and hospitality.

This work is partly supported by the grant of the President of Russian Federation (NS1616.2008.2), the RFBR grant 08-02-00473 and by the grant of Dynasty Foundation awarded by the Scientific Board of ICFPM.

\section{A Appendix}

In this Appendix we give detailed derivations of the dispersion relations $(4.24)-(4.29)$ for the fermionic spectrum. For simplicity we introduce the following dimensionless variables $t=p / k, \varepsilon=E / k$, and $\mu=m / k$. We start from the equation (4.20).

Small momenta. Let us firstly discuss the regime of small momenta $t \ll 1$. One can expand the l.h.s. of $(4.20)$ at small $t$ and obtain the following equation

$$
\left[1+\frac{t}{2 \nu-1}+\frac{\Gamma(1 / 2-\nu)}{\Gamma(1 / 2+\nu)}\left(\frac{t}{2}\right)^{2 \nu}\right]=\gamma \frac{\mu-\alpha \varepsilon}{\nu}\left[1-\frac{t}{2 \nu-1}-\frac{\Gamma(1 / 2-\nu)}{\Gamma(1 / 2+\nu)}\left(\frac{t}{2}\right)^{2 \nu}\right] .
$$

Here we kept two terms in the next to the leading order since depending on $\nu$ these terms may compete with each other; the corrections to the equation are of the order of $t^{2}$ and $t^{2 \nu+1}$. If $\operatorname{Re} \nu \neq 0$ (which automatically implies $\mu \neq 0$ ) then the leading order is represented by the first terms in the square brackets in (A.1). Thus in order to satisfy the equation one is forced to conclude that $\varepsilon \rightarrow 0$ as $t \rightarrow 0$ and $\gamma=1$, which means that we deal with the zero mode. Aside from that, it turns out that $\alpha$ is negative. This fact follows straightforwardly from $(\overline{4.20})$. Indeed, the operator $(4.11)$ with the boundary conditions under consideration 
is hermitian, so, if $\operatorname{Re} \nu \neq 0$ then $\operatorname{Im} \nu=0$. It is well known that at real values of order the modified Bessel function $\mathrm{K}_{\nu}(t)$ is positively defined and at fixed argument is an increasing function of $\nu$. Thus, $\xi_{-}(t)>\xi_{+}(t)>0$ what means that $\alpha=-1$ and $\gamma=1$.

In order to find the behavior of the zero mode at $t \rightarrow 0$ one needs to take into account subleading terms in (A.1). Expanding $(\overline{A .1})$ at small $\epsilon$ and $t$ one obtains

$$
\epsilon \simeq \frac{2 \mu}{2 \mu-1} t+2 \mu \frac{\Gamma\left(\frac{1}{2}-\mu\right)}{\Gamma\left(\frac{1}{2}+\mu\right)}\left(\frac{t}{2}\right)^{2 \mu} .
$$

which reconstructs all dispersion relations for zero mode $(\overline{4.24})-(\overline{4.26})$ at small momenta. Indeed, if $\mu>1 / 2$ then the first term in $(\mathrm{A} .2)$ is dominant, the second term is dominant in the opposite case $(\mu<1 / 2)$. In the case $\mu \rightarrow 1 / 2$ both terms tend to infinity becoming comparable. Expanding the r.h.s. of $(\mathrm{A} .2)$ at $\mu=1 / 2$ one easily verifies that divergent parts are cancelled and obtains (4.25).

Recall that in the above analysis we assumed $\operatorname{Re} \nu \neq 0$. Let us now consider the case when $\nu$ is pure imaginary. In this case the leading terms in the brackets in (A.1) are the first and third one. Then after some calculations one obtains from (A.1) the following equation (the same equation follows directly from $(4.16)$ )

$$
\left(\frac{t}{2}\right)^{-2 \nu} \Gamma\left(\frac{1}{2}+\nu\right)^{2}(\gamma \mu-\nu)-\left(\frac{t}{2}\right)^{2 \nu} \Gamma\left(\frac{1}{2}-\nu\right)^{2}(\gamma \mu+\nu)=0 .
$$

which is equivalent to

$$
-2 \nu \log \frac{t}{2}+2 i \arctan \frac{1}{i} \frac{\Gamma(1 / 2+\nu)-\Gamma(1 / 2-\nu)}{\Gamma(1 / 2+\nu)+\Gamma(1 / 2-\nu)}+i \arctan \left[\gamma \frac{i \nu}{\mu}\right]=\pi n i,
$$

where $\mathrm{n}$ is an integer. Since the last two terms in the l.h.s. of this equation are finite at all values of imaginary $\nu$, the only way to satisfy the equation at fixed $n$ is to assume that $\nu$ thends to zero as $t \rightarrow 0$. Note, however, that the expression $(\overline{A .4})$ does not depend on $\alpha$, i.e. it holds for both helicities. In order to clarify the dependence on $\alpha$ one should find $\nu$ directly from (A.1) (the advantage of (A.3) is that this equation allows one to establish quite easily the fact that $\nu \rightarrow 0$ as $t \rightarrow 0$, while it is not so obvious from (A.1)).

Performing small $\nu$ expansion in (A.1) one obtains

$$
\tanh \left(\nu \Psi\left(\frac{1}{2}\right)-\nu \log \frac{t}{2}\right)=\left(\frac{2 \gamma \mu}{\nu}\right)^{\alpha}
$$

This equation tells us that if $\alpha=1$ then the argument of tanh should go to $i \pi\left(l+\frac{1}{2}\right), l \in \mathbb{Z}$, meanwhile if $\alpha=-1$ the argument tends to $i \pi l$. Then the solution of (A.5) can be written as follows

$$
\nu_{n}(t)=\frac{i \pi n}{2 \Psi\left(\frac{1}{2}\right)-\frac{\gamma}{\mu}-2 \log \frac{t}{2}},
$$

where $n \in \mathbb{N}$ (recall that $\nu \neq 0$ and we assume that $\operatorname{Im}(\nu)>0$ ), and mode helicities depend on the level number as $\alpha=(-1)^{n+1}$. The above equation in turn gives us the dispersion 
relation for the heavy modes $(4.27)$. Note that $(\overline{\text { A.6 }})$ is obtained in the assumption $|\nu| \ll 1$. Thus for $n$ large enough the momentum $t$ should be small. However, it does not affect the conclusion that the spectrum is discrete and $E_{n} \rightarrow m$ as $t \rightarrow 0$.

Let us now consider the case $m_{\psi}=m$, i.e. we start from (4.21). Comparing (4.21) with (4.20) one sees that (A.1) still holds if one replaces the factor $(\mu-\alpha \varepsilon) / \nu$ in the r.h.s. by the factor of $i$ which is equivalent to taking $\mu=0$ and $\alpha=1$ in this (and only in this) factor. The equation (A.3) also holds if one puts $\gamma \mu=0$ in the parentheses. So the conclusion that $\nu$ goes to zero as $t \rightarrow 0$ remains intact, i.e. all modes have the same mass $\mu$, in particular in the massless case all modes become gapless. In the regime $\nu \rightarrow 0$ one can obtain from (A.1) the following equation (an analog of (A.5))

$$
\tanh \left(\nu \Psi\left(\frac{1}{2}\right)-\nu \log \frac{t}{2}\right)=-i \gamma
$$

Then

$$
\nu_{n}(t)=\frac{i \pi(2 n+1)}{4 \Psi\left(\frac{1}{2}\right)-4 \log \frac{t}{2}} .
$$

Note that $\gamma$ depends on the mode number $n$ as $\gamma=(-1)^{n+1}$. From (A.7) the dispersion relation (4.28) immediately follows.

High momenta. Let us now consider the high momenta regime $t \gg \mu, \epsilon$. In this case it is easy to start from (4.11) and neglect the last term compared with the term proportional to $p^{2}$. Then the solutions to the equations of motion reads

$$
\xi_{ \pm}=\mathrm{K}_{\nu}(t), \quad \nu=\sqrt{\mu^{2}-\varepsilon^{2}},
$$

and the equation yielding the dispersion relation (an analog of $(4.16)$ ) is the following

$$
t \mathrm{~K}_{\nu}^{\prime}(t)=0
$$

where we neglected the term $\mu \mathrm{K}_{\nu}$ which appears in the case $m_{\psi}=m \cdot \operatorname{sign}(z)$. Thus one can consider both cases $\left(m_{\psi}=m \cdot \operatorname{sign}(z)\right.$ and $\left.m_{\psi}=m\right)$ on an equal footing.

There are three different possibilities in the regime $t \rightarrow \infty: 1)|\nu| \ll t, 2)|\nu|>t, 3$ ) $|\nu| \rightarrow \infty$ but $|\nu|<t$. In the first case one can use the asymptotic behavior of the Bessel function with large argument and fixed order

$$
\mathrm{K}_{\nu}(t)=\sqrt{\frac{\pi}{2 t}} \mathrm{e}^{-t}\left(1+\mathcal{O}\left(t^{-1}\right)\right) .
$$

Due to the fact that the leading order in (A.9) does not depend on $\nu$ there is no solution of (A.8) in this case. In the second and in the third cases $\varepsilon \rightarrow \infty$ as $t \rightarrow \infty$, so $\nu$ is pure imaginary $\nu=i|\nu|$. In the second case one uses the asymptotic expansion for the Bessel function $[15$

$$
\mathrm{K}_{i|\nu|}(t)=\frac{\sqrt{2}}{\sqrt[4]{|\nu|^{2}-t^{2}}} \mathrm{e}^{\frac{|\nu| \pi}{2}}\left[\Gamma\left(\frac{1}{2}\right) \sin \left(|\nu| \operatorname{Arccosh} \frac{|\nu|}{t}-\sqrt{|\nu|^{2}-t^{2}}+\frac{\pi}{4}\right)+\mathcal{O}\left(t^{-1}\right)\right] .
$$


Substituting this expression into $(\mathrm{A} .8)$ one obtains the equation

$$
\tan \left(|\nu| \operatorname{Arccosh} \frac{|\nu|}{t}-\sqrt{|\nu|^{2}-t^{2}+\frac{\pi}{4}}\right)=2 \frac{\left(|\nu|^{2}-t^{2}\right)^{\frac{3}{2}}}{t^{2}} .
$$

The solution of this equation reads

$$
|\nu|=t+\mathcal{O}\left(t^{1 / 3}\right)
$$

where the correction is positive, so the condition $|\nu|>t$ is satisfied. From this equation one obtains (4.29).

In the last case $|\nu|<t$ one uses the asymptotic expansion $[15$

$$
\mathrm{K}_{i|\nu|}=\frac{\Gamma\left(\frac{1}{2}\right)+\mathcal{O}\left(t^{-1}\right)}{\sqrt[4]{4\left(t^{2}-|\nu|^{2}\right)}} \exp \left(-\sqrt{t^{2}-|\nu|^{2}}-|\nu| \arcsin \frac{|\nu|}{t}\right) .
$$

Substituting this expansion in $(\overline{\mathrm{A} .8})$ one again ends up with the solution $(\mathrm{A} .10)$ where the correction is positive as well. It contradicts however the assumption $|\nu|<t$. Thus there is no solution of $(\mathrm{A} .8)$ in this case.

\section{References}

[1] P. Koroteev and M. Libanov, "On Existence of Self-Tuning Solutions in Static Braneworlds without Singularities", JHEP 0802, 104 (2008), 0712.1136

[2] V. A. Rubakov and M. E. Shaposhnikov, "Do We Live Inside a Domain Wall?", Phys. Lett. B125, 136 (1983). • N. Arkani-Hamed, S. Dimopoulos and G. R. Dvali, "The hierarchy problem and new dimensions at a millimeter", Phys. Lett. B429, 263 (1998), hep-ph/9803315.

[3] L. Randall and R. Sundrum, "A large mass hierarchy from a small extra dimension", Phys. Rev. Lett. 83, 3370 (1999), hep-ph/9905221.

[4] L. Randall and R. Sundrum, "An alternative to compactification", Phys. Rev. Lett. 83, 4690 (1999), hep-th/9906064.

[5] V. A. Rubakov and M. E. Shaposhnikov, "Extra Space-Time Dimensions: Towards a Solution to the Cosmological Constant Problem", Phys. Lett. B125, 139 (1983).

I. Antoniadis, N. Arkani-Hamed, S. Dimopoulos and G. R. Dvali, "New dimensions at a millimeter to a Fermi and superstrings at a TeV", Phys. Lett. B436, 257 (1998), hep-ph/9804398. • R. Sundrum, "Compactification for a three-brane universe", Phys. Rev. D59, 085010 (1999), hep-ph/9807348 • B. Bajc and G. Gabadadze, "Localization of matter and cosmological constant on a brane in anti de Sitter space",

Phys. Lett. B474, 282 (2000), hep-th/9912232. • N. Arkani-Hamed, S. Dimopoulos, N. Kaloper and R. Sundrum, "A small cosmological constant from a large extra dimension", Phys. Lett. B480, 193 (2000), hep-th/0001197. • C. Csaki, J. Erlich, C. Grojean and T. J. Hollowood, "General properties of the self-tuning domain wall approach to the cosmological constant problem", Nucl. Phys. B584, 359 (2000), hep-th/0004133. • G. Dvali, G. Gabadadze and M. Shifman, "Diluting cosmological constant in infinite volume extra 
dimensions", Phys. Rev. D67, 044020 (2003), hep-th/0202174. • U. Gunther, P. Moniz and A. Zhuk, "Nonlinear multidimensional cosmological models with form fields: Stabilization of extra dimensions and the cosmological constant problem", Phys. Rev. D68, 044010 (2003),

hep-th/0303023. • C. P. Burgess, "Supersymmetric large extra dimensions and the cosmological constant problem", hep-th/0510123. • C. P. Burgess, "Extra Dimensions and the Cosmological Constant Problem", 0708.0911. • S. M. Carroll and M. M. Guica, "Sidestepping the cosmological constant with football- shaped extra dimensions", hep-th/0302067.

[6] E. Witten, "The cosmological constant from the viewpoint of string theory", hep-ph/0002297.

[7] P. Binetruy, C. Deffayet and D. Langlois, "Non-conventional cosmology from a brane-universe", Nucl. Phys. B565, 269 (2000), hep-th/9905012. • P. Binetruy, C. Deffayet, U. Ellwanger and D. Langlois, "Brane cosmological evolution in a bulk with cosmological constant", Phys. Lett. B477, 285 (2000), hep-th/9910219.

[8] T. G. Rizzo, "More and more indirect signals for extra dimensions at more and more colliders", Phys. Rev. D59, 115010 (1999), hep-ph/9901209. • ATLAS Collaboration, A. Gupta, "Search for extra space dimensions with ATLAS", Pramana 62, 607 (2004). ATLAS Collaboration, L. Vacavant, "Search for extra dimensions at LHC", Eur. Phys. J. C33, s924 (2004), hep-ex/0310020. • K. Benslama, "Search for extra dimensions with ATLAS at LHC", Prepared for Lake Louise Winter Institute 2004 on Fundamental Interactions (LL WI 2004), Lake Louise, Alberta, Canada, 15-21 Feb 2004. • A. A. Pankov, "Probing and identifying extra dimensions at LHC", Nonlin. Phenom. Complex Syst. 7, 385 (2004). • E. W. Dvergsnes, P. Osland, A. A. Pankov and N. Paver, "Search and identification of extra spatial dimensions at LHC", Int. J. Mod. Phys. A20, 2232 (2005), hep-ph/0410402. • A. Ghezzi, "Search for extra dimensions at the LHC", AIP Conf. Proc. 794, 146 (2005). • ATLAS Collaboration, F. M. Brochu, "Search for extra-dimensions in the ATLAS experiment", Czech. J. Phys. 55, B643 (2005). • D. Dannheim, "Probing extra dimensions with ATLAS", AIP Conf. Proc. 903, 261 (2007), hep-ex/0611005. • K. Agashe, A. Belyaev, T. Krupovnickas, G. Perez and J. Virzi, "LHC signals from warped extra dimensions", Phys. Rev. D77, 015003 (2008), hep-ph/0612015. • S. Shmatov, "Search for Extra Dimensions with Atlas and CMS Detectors at the LHC", 0707.0470. • D. Berry, Tracey, "Sensitivity of the LHC Experiments to Extra Dimensions", 0710.4887. - H. Melbeus and T. Ohlsson, "Searches for hyperbolic extra dimensions at the LHC", 0806.1841.

[9] K. Agashe, N. G. Deshpande and G. H. Wu, "Can extra dimensions accessible to the SM explain the recent measurement of anomalous magnetic moment of the muon?", Phys. Lett. B511, 85 (2001), hep-ph/0103235. • EOT-WASH Group Collaboration, E. G. Adelberger, "Sub-millimeter tests of the gravitational inverse square law", hep-ex/0202008. • J. C. Long and J. C. Price, "Current short-range tests of the gravitational inverse square law", Comptes Rendus Physique 4, 337 (2003), hep-ph/0303057. • J. M. Frere, M. V. Libanov, E. Y. Nugaev and S. V. Troitsky, "Searching for family-number conserving neutral gauge bosons from extra dimensions", JETP Lett. 79, 598 (2004), hep-ph/0404139.

[10] V. A. Rubakov, "Large and infinite extra dimensions: An introduction", Phys. Usp. 44, 871 (2001), hep-ph/0104152. • G. Gabadadze, "ICTP lectures on large extra dimensions", hep-ph/0308112. • C. Csaki, "TASI lectures on extra dimensions and branes", hep-ph/0404096. • P. Brax, C. van de Bruck and A.-C. Davis, "Brane world cosmology", Rept. Prog. Phys. 67, 2183 (2004), hep-th/0404011. 
[11] T. G. Rizzo, "Lorentz violation in extra dimensions", JHEP 0509, 036 (2005), hep-ph/0506056. • V. Baukh, A. Zhuk and T. Kahniashvili, "Extra dimensions and Lorenz invariance violation", Phys. Rev. D76, 027502 (2007), 0704.0314. • D. S. Gorbunov and S. M. Sibiryakov, "Ultra-large distance modification of gravity from Lorentz symmetry breaking at the Planck scale", JHEP 0509, 082 (2005), hep-th/0506067. • D. S. Gorbunov and S. M. Sibiryakov, "Self-accelerated brane Universe with warped extra dimension", 0804.2248 .

[12] S. L. Dubovsky, "Tunneling into extra dimension and high-energy violation of Lorentz invariance", JHEP 0201, 012 (2002), hep-th/0103205.

[13] M. V. Libanov and V. A. Rubakov, "Lorentz-violation and cosmological perturbations: A toy brane-world model", JCAP 0509, 005 (2005), astro-ph/0504249. • M. V. Libanov and V. A. Rubakov, "Lorentz-violating brane worlds and cosmological perturbations", Phys. Rev. D72, 123503 (2005), hep-ph/0509148. • F. Ahmadi, S. Jalalzadeh and H. R. Sepangi, "Lorentz violation in brane cosmology, accelerated expansion and fundamental constants", Class. Quant. Grav. 23, 4069 (2006), gr-qc/0605038

[14] A. E. Nelson, "A new angle on intersecting branes in infinite extra dimensions", Phys. Rev. D63, 087503 (2001), hep-th/9909001. • G. R. Dvali, G. Gabadadze and M. Porrati, "Metastable gravitons and infinite volume extra dimensions", Phys. Lett. B484, 112 (2000), hep-th/0002190.

[15] H. Bateman and A. Erdélyi, "Higher Transcendental Functions, vol. 2", McGraw-Hill Book Co. (1954), New York.

[16] T. Gherghetta and A. Pomarol, "Bulk fields and supersymmetry in a slice of AdS", Nucl. Phys. B586, 141 (2000), hep-ph/0003129. • Y. Grossman and M. Neubert, "Neutrino masses and mixings in non-factorizable geometry", Phys. Lett. B474, 361 (2000), hep-ph/9912408. • T. Gherghetta, "Warped models and holography", hep-ph/0601213.

[17] Y.-X. Liu, L.-D. Zhang, L.-J. Zhang and Y.-S. Duan, "Fermions on Thick Branes in Background of Sine-Gordon Kinks", 0804.4553.

[18] E. Witten, "FERMION QUANTUM NUMBERS IN KALUZA-KLEIN THEORY", Lecture given at Shelter Island II Conf., Shelter Island, N.Y., 1-2 Jun 1983. • D. Bailin and A. Love, "KALUZA-KLEIN THEORIES", Rept. Prog. Phys. 50, 1087 (1987).

[19] S. Kachru, X. Liu and M. Mulligan, "Gravity Duals of Lifshitz-like Fixed Points", Phys. Rev. D78, 106005 (2008), 0808.1725.

[20] I. Gordeli and P. Koroteev, "Comments on Holography with Broken Lorentz Invariance", 0904.0509 . 